\title{
Outer membrane VDAC1 controls permeability transition of the inner mitochondrial membrane in cellulo during stress-induced apoptosis
}

Flora Tomasello ${ }^{1,2}$, Angela Messina ${ }^{2,3}$, Lydia Lartigue ${ }^{1}$, Laura Schembri ${ }^{1}$, Chantal Medina ${ }^{1}$, Simona Reina ${ }^{2,3}$, Didier Thoraval ${ }^{4}$, Marc Crouzet ${ }^{4}$, François Ichas ${ }^{1,5}$, Vito De Pinto ${ }^{2,3}$, Francesca De Giorgi ${ }^{1,5}$

${ }^{I}$ INSERM U916, Université Bordeaux 2, Institut Bergonié, 229 cours de l'Argonne, 33076 Bordeaux, France; ${ }^{2}$ Dipartimento Scienze Chimiche, Università di Catania, Catania, Italia; ${ }^{3}$ Istituto Nazionale di Biomembrane e Biosistemi, Sezione di Catania, Roma, Italia; ${ }^{4}$ CNRS UMR 5095, Université Bordeaux 2, 146 rue Leo Saignat, 33076 Bordeaux, France; ${ }^{5}$ FluoFarma, 2 rue Robert Escarpit, 33600 Pessac, France

Voltage-dependent anion channel (VDAC)1 is the main channel of the mitochondrial outer membrane (MOM) and it has been proposed to be part of the permeability transition pore (PTP), a putative multiprotein complex candidate agent of the mitochondrial permeability transition (MPT). Working at the single live cell level, we found that overexpression of VDAC1 triggers MPT at the mitochondrial inner membrane (MIM). Conversely, silencing VDAC1 expression results in the inhibition of MPT caused by selenite-induced oxidative stress. This MOM-MIM crosstalk was modulated by Cyclosporin A and mitochondrial Cyclophilin D, but not by Bcl-2 and Bcl- $X_{L}$, indicative of PTP operation. VDAC1-dependent MPT engages a positive feedback loop involving reactive oxygen species and p38-MAPK, and secondarily triggers a canonical apoptotic response including Bax activation, cytochrome $c$ release and caspase 3 activation. Our data thus support a model of the PTP complex involving VDAC1 at the MOM, and indicate that VDAC1-dependent MPT is an upstream mechanism playing a causal role in oxidative stress-induced apoptosis.

Keywords: apoptosis, mitochondria, VDAC, MPT, PTP, oxidative stress, selenite

Cell Research (2009) 19:1363-1376. doi: 10.1038/cr.2009.98; published online 11 August 2009

Correspondence: Vito De Pinto ${ }^{a}$, Francesca De Giorgi ${ }^{\mathrm{b}}$

${ }^{a}$ Tel: +39 095 7385138/371155; Fax: +39095 580138/371155

E-mail:vdpbiofa@unict.it

bE-mail: francesca.degiorgi-ichas@inserm.fr

Abbreviations: MAPK (mitogen-activated protein kinase); cyt $c$ (cytochrome $c$ ); NAC (N-acetyl-Cysteine); FMK (fluoromethyl ketone); z-VADFMK (Val-Asp(OCH3)-z-Asp(OCH3)-FMK); HEPES (4-(2-Hydroxyethyl) piperazine-1-ethanesulfonic acid); PBS (phosphate-buffered saline); STS (staurosporine); FCCP (carbonylcyanide-p-trifluoromethoxyphenyl hydrazone); TMRM (tetramethylrhodamine methylester); DCFH-DA (dichlorofluorescein diacetate); FITC (fluorescein isothiocyanate); GFP (green fluorescent protein); dsRed (Discosoma red fluorescent protein); CFP (cyan fluorescent protein); YFP (yellow fluorescent protein); ROS (reactive oxygen species); siRNA (small inhibitory RNAs); CsA (cyclosporin A); CyP-D (cyclophilin D); VDAC (voltage-dependent anion channel); ANT (adenine nucleotide translocator); $\triangle \Psi \mathrm{m}$ (mitochondrial transmembrane potential); MOMP (mitochondrial outer membrane permeabilization); PTP (permeability transition pore); MOM (mitochondrial outer membrane); MIM (mitochondrial inner membrane); MPT (mitochondrial permeability transition); IMS (inner membrane space); TNF $\alpha$ (tumor necrosis factor alfa); Pp38 (phosphorylated p38 kinase)

Received 5 February 2009; revised 25 May 2009; accepted 24 June 2009; published online 11 August 2009

\section{Introduction}

The mitochondrial inner membrane (MIM) can undergo a sudden increase in permeability to ions and small solutes that then equilibrate across the membrane while the macromolecules $>1500$ Da remain trapped in the mitochondrial matrix. This sudden change is called the mitochondrial permeability transition (MPT) and is accompanied by an immediate collapse of the mitochondrial membrane potential $(\Delta \psi)$. The resulting osmotic imbalance leads - at least in vitro - to mitochondrial matrix swelling and rupture of the mitochondrial outer membrane (MOM) [1]. While the conditions capable of causing the latter osmotic process are probably never met in cellulo, under specific conditions, MPT can, however, be followed by the release of cytochrome $c$ (cyt $c$ ) and other mitochondrial proteins into the cytoplasm and apoptosis [2]. MPT is thought to be mediated by the opening of a putative voltage-dependent high-conductance mega- 
channel called the permeability transition pore (PTP) [3].

Based on several biochemical and functional studies, a general consensus was reached that the PTP is a multicomponent protein complex. The PTP complex would assemble at contact sites between the MIM and the $\mathrm{MOM}$ as a consequence of interactions between VDAC1 (voltage-dependent anion channel), ANT (adenine nucleotide translocase) and Cyp-D (cyclophilin D) [4]. However, mitochondria from knockout mice lacking the ANT, Cyp-D or VDAC can all undergo MPT [5]. Hence, alternative models have been proposed where the MPT is not caused by a PTP complex constituted of defined proteins, but instead results from unspecific interactions between mitochondrial membrane proteins $[6,7]$. Alternatively, Cyp-D may act as a "PTP-organizing centre" for other mitochondrial proteins spanning the mitochondrial membranes such as solute carriers or constituents of the protein import machinery.

VDACs, also known as mitochondrial porins, are the most abundant integral membrane proteins found in the MOM. They form pores that are permeable to hydrophilic solutes up to $3000 \mathrm{Da}$ [8-10]. The structure of VDAC1, the most abundant isoform, has recently been solved by NMR and X-ray crystallography [11-13]. VDAC1 presents in reconstituted systems the following two main conductance states depending on the voltage applied: a slightly anion-selective open state that allows the diffusion of large metabolites including nucleotides and a cation-selective low-conductance closed state that controls the ATP flux through the membrane [14]. It is not clear what shifts VDAC1 between the two states in vivo.

Some models of MOM permeabilization during apoptosis consider that VDAC1 is a key player in this process. According to Tsujimoto and co-workers $[15,16]$, in physiological conditions, VDAC1 prominently exists in a low-conductance state that allows the exchange of metabolites between the mitochondria and cytosol. Upon apoptosis induction, VDAC1 exhibits an increased conductance associated with PTP opening, dissipation of $\Delta \Psi$, efflux of inter membrane space (IMS) proteins and cell death. In contrast, Thompson and colleagues [17] propose that VDAC1 exists normally in a high-conductance state and shifts to closed state in pro-apoptotic conditions. They postulate that VDAC1 closure leads to a transient hyper-polarization driving the influx of charged solutes and water, thus causing matrix swelling, MOM rupture, release of IMS proteins and cell death [18].

Interestingly, VDAC has been reported to bind to the pro- and anti-apoptotic proteins of the Bcl-2 family that regulate the permeability of the $\operatorname{MOM}[18,19]$. It may interact with mitochondrial proteins involved in the formation of contact sites between the inner and outer membranes [20]. It may also bind to cytosolic proteins, such as hexokinase I and hexokinase II [21-23], or to cytoskeletal proteins like actin [24], gelsolin [25] and tubulin [26]. These ligands have been proposed to influence the response of VDAC1 to Bcl-2 family proteins and thereby modulate its role in apoptosis [27].

VDAC1 expression levels are finely tuned in the cell. Downregulation by silencing dramatically slows cell growth and decreases ATP production in human T-REx293 cells [28]. VDAC1 overexpression induces apoptotis in animal, worm and vegetal cells, suggesting that VDAC is part of the mitochondrial death machinery [2932].

Selenium is an essential micronutrient, which, upon conversion to the amino acid selenocysteine, enters selenoproteins including glutathione peroxidase and thioredoxin reductase [33]. At micromolar concentrations, sodium selenite induces oxidative stress [34, 35], and induces PTP opening, making this element one of the most potent inducers of MPT. Concentrations of 0.1-10 $\mu \mathrm{M}$ selenite are sufficient to produce cyclosporin A (CsA)sensitive mitochondrial $\Delta \Psi$ collapse in living cells [36].

Although the notion that VDAC1 might somehow be involved in cell death is a recurrent theme in the apoptosis literature, the molecular mechanism of VDAC1 regulation and its participation in the PTP complex are still debated. In this work, we provide evidence that VDAC1 functionally participates in the PTP complex in cellulo. Moreover, we found that upon VDAC1 depletion, cells became specifically insensitive to the oxidative stress inducer sodium selenite but not to staurosporine (STS), thus indicating that VDAC1 underlies the sensitivity of the PTP complex to reactive oxygen species (ROS).

Our observations thus indicate that (i) VDAC participates in the PTP complex in cellulo and (ii) it is a ROS sensor [37] that triggers opening of the PTP complex under conditions of oxidative stress [2, 34, 38-40] but not during "physiological" apoptosis mimicked by STS [41].

\section{Results}

Overexpression of VDAC1 triggers PTP operation and signals cell death through $\mathrm{p} 38$ kinase activation

To test the possible participation of VDAC1 in MPT in cellulo, we transientlty overexpressed it in COS7 cells as a HA-tagged protein using the double promoter reporter vector pCMS-GFP. Under those conditions, VDAC1 is overexpressed (Figure 1B) and correctly sorted to the MOM of mitochondria (Figure 1A). Since the expression of VDAC1-HA (immunofluorescence with anti-HA, Figure 1B) was always associated with the expression 
of green fluorescent protein (GFP), we used the GFP signal as a transfection marker to perform analysis on individual living cells. To detect MPT, we monitored $\Delta \Psi$ with the Nernstian dye tetramethylrhodamine methylester (TMRM) in living cells overexpressing VDAC1 (48 $\mathrm{h}$ post-transfection, GFP-positive cells). Measurement of GFP and TMRM $(\Delta \Psi)$ signals by flow cytometry showed that over $60 \%$ of the cells overexpressing VDAC1 exhibited a collapsed $\Delta \Psi$ (Figure 1C), compatible with the idea that upregulation of VDAC1 triggers MPT. The loss of $\triangle \Psi$ observed in VDAC1-transfected cells was specific for VDAC1. Transfection of proteins targeted to other locations in the cell failed to induce $\Delta \Psi$ changes (not shown). Notably, to test whether the loss of $\Delta \Psi$ induced by VDAC1 could simply be due to an "overcrowding effect" resulting in non-specific MOM destabilization, we overexpressed an artificial integral outer mitochondrial membrane protein (MOM-GFP) [42] and found no significant MPT induction (Figure 1D).

Further, the PTP-dependence of the observed effect was suggested by the fact that concomitant overexpression of Cyp-D, which is also a candidate subunit of the PTP complex and regulates channel closure [43], efficiently antagonized MPT induced by VDAC1 upregulation (Figure 1D). These findings suggest that the $\Delta \Psi$ collapse that accompanies VDAC1-induced MPT is a direct consequence of the operation of the PTP complex rather than a downstream event dependent on effector caspases [44]. This conclusion is supported by the following experimental evidence: (i) expression of VDAC1 in POR1-deleted yeast cells elicits MPT in the absence of any Bcl-2 family protein or effector caspases (data not shown), (ii) neither Bcl-2 nor Bcl-XL could prevent $\Delta \Psi$ dissipation by VDAC1 (Figure 1D) and (iii) neither the pan-caspase inhibitor zVADfmk nor the XIAP protein could prevent VDAC1-induced MPT, while CsA, a reference PTP inhibitor, counteracted the effects of VDAC1 upregulation (Figure 1E). Remarkably pre-treatment with either the antioxidant $\mathrm{N}$-acetyl-Cysteine (NAC) or the p38 inhibitor SB203580 [45] strongly reduced the $\Delta \Psi$ loss induced by VDAC1 overexpression (Figure 1E), suggesting the involvement of ROS and MAP kinases in a positive feedback loop aggravating MPT.

To unequivocally assess the role of ROS in hVDAC1induced apoptosis, we measured the intracellular ROS production by monitoring the increase in dichlorofluorescein diacetate (DCFH-DA) dye fluorescence in living cells by confocal microscopy. An increase in DCFH-DA fluorescence intensity was observed approximately $18 \mathrm{~h}$ post transfection in cells overexpressing hVADC1. The DCFH-DA fluorescence decreased after $30 \mathrm{~h}$ of VDAC1 overexpression, probably as a consequence of the acti- vation of intracellular ROS scavengers (Figure 2A). In contrast, control cells maintained a relatively stable, low ROS production throughout the experimental period ( 30 h). The increase in intracellular ROS levels induced by hVDAC1 overexpression was completely abrogated by pre-treatment with NAC (Figure 2A).

VDAC1-induced MPT committed HeLa cells to apoptotic death. Following $\Delta \Psi$ collapse induced by VDAC1, Bax was activated and relocated to the MOM in $80 \%$ of the cells (Figure 2C). Concurrent with Bax activation, a similar fraction of transfected cells showed diffuse cyt $c$ staining (Figure 2D).

Caspase activation is a fundamental component in the mammalian apoptotic process. Caspase 3 is one of the main executioner caspases activated during apoptosis, and this activation can be used as an apoptotic marker. Caspase 3 activation was assessed in living cells with Caspatag $^{\circledR}$. Caspase 3 was activated in cells overexpressing VDAC1 for $50 \mathrm{~h}(50 \%$ vs $18 \%$ in control cells, $P<$ $0.01)$. A similar level of caspase activation was reached after challenging cells with the apoptotic inducers, STS and selenite (Figure 2B).

Taken together, these findings indicate that the MPT triggered by VDAC1 upregulation is mediated by ROS generation and triggers a canonical sequence of apoptotic signaling events marked by an early p38-dependent positive feedback loop.

Sodium selenite induces cell death via PTP operation and 38 kinase activation

To further test the possible participation of VDAC1 in the PTP, we investigated the signaling pathway triggered by sodium selenite, which is known to trigger PTP opening in cellulo [34-36]. Time-course experiments indicated that $73 \%$ of HeLa cells incubated with $20 \mu \mathrm{M}$ sodium selenite underwent $\Delta \Psi$ collapse after a 3 -h exposure (Figure $3 \mathrm{~A})$. Kinetic measurements of Bax translocation and cyt $c$ release were performed with clones stably expressing GFP-Bax (Cl10) or Cyt $c$-GFP (2H18). These experiments revealed that in most cells $(70 \%)$, Bax relocates to the MOM and cyt $c$ diffuses into the cytosol with a delay of about $3 \mathrm{~h}$ with respect to PTP opening (Figure $3 \mathrm{~A}$ ). Pre-incubation with $10 \mu \mathrm{M}$ CsA efficiently prevented selenite-mediated mitochondrial depolarization (Figure 3B), confirming that selenite directly stimulates PTP opening. In agreement with the notion that selenite exerts its pro-apoptotic effects by oxidizing thiol-containing cellular substrates and generating ROS [36, 46, 47], we found that intracellular ROS increased within $30 \mathrm{~min}$ after selenite addition, reached the maximum value at $45 \mathrm{~min}$ and then returned to the basal level (Figure 3C). This transient ROS increase appeared to be the cause of 


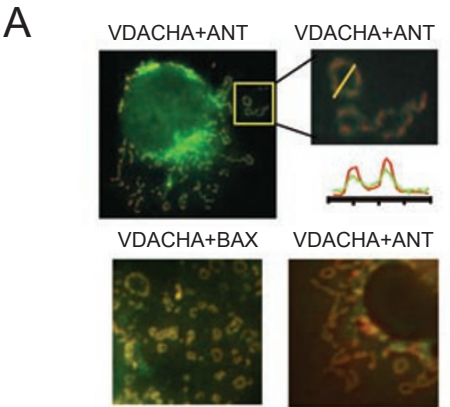

C
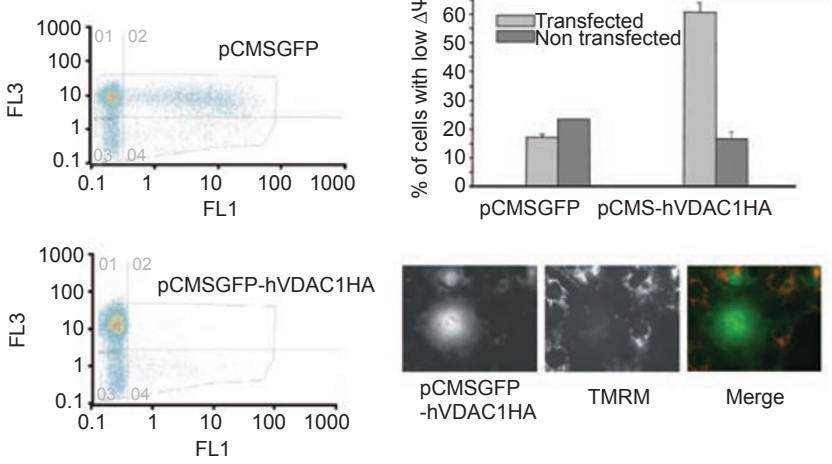

B
HeLa pCMShVDAC

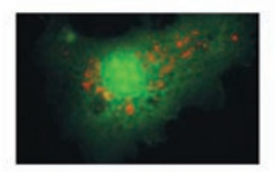

HeLa pCMShVDAC1

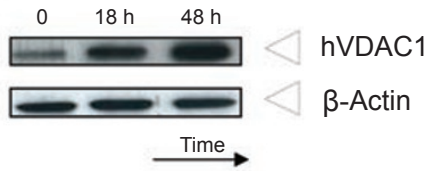

$\mathrm{D}$

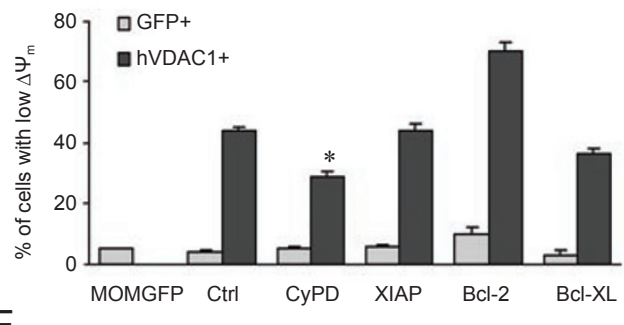

$\mathrm{E}$

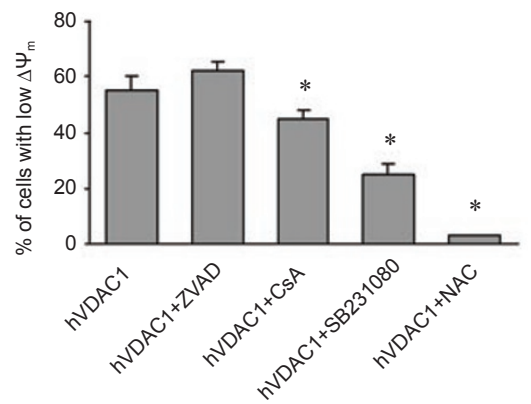

Figure 1 VDAC1 overexpression triggers the PTP opening via ROS increase and p38 activation. (A) Subcellular localization of recombinant overexpressed hVDAC1-HA. The upper panel shows the immunofluorescence of the HA (VDAC, red) and the endogenous mitochondrial protein ANT2 (green) in COS7 cells transfected with pIRES-hVADC1HA. The right panel is a magnification of the indicated area. Fluorescence distribution analysis indicates the colocalization of recombinant hVDAC1HA and endogenous ANT2 on a single mitochondrion. Two more double immunofluorescences are shown in the lower panel. Left: hVDAC1-HA (red) colocalizes with activated mitochondrial Bax (green) in the MOM; Right: the hVDAC1-HA signal (green) in the MOM encircles the ANT2 signal (red) in the MIM. (B) pCMS-GFP-hVDAC1-HA transfection results in a simultaneous expression of both mitochondrial hVDAC1-HA (red, anti-HA) and cytosolic GFP (green). The western blot in the right panel shows the time-dependent increase of the hVDAC1 levels in cells transfected with pCMS-GFP-hVDAC1-HA. Total lysates from HeLa cells transfected or not with pCMS-GFP-hVADC1HA were immunoblotted by using an anti-VDAC1 antibody. $\beta$-Actin served as loading and transfer efficiency control. (C) Effect of hVDAC1 upregulation on mitochondrial $\triangle \Psi$. COS-7 cells were transfected with pCMS-GFP-VDAC1-HA or with the control pCMS-GFP, incubated with TMRM to monitor $\Delta \Psi$ and then examined either under an inverted fluorescent microscope or by flow cytometry. Left panels: representative flow cytometric dot plots of GFP or GFP+VDAC1 transfected cells stained with TMRM. Upper right panel: quantification of the fraction of cells with collapsed $\Delta \Psi$ among the GFP-positive and negative cells (fraction of cells with low FL3 signal and high FL1 signal). Bars: SE, * indicates a significant difference $\left(P<0.001, \chi^{2}\right.$-test). Lower right panel: representative image of pCMS-GFPVDAC1-HA transfected cells loaded with TMRM showing a GFP/VDAC1 positive cell that failed to take up TMRM due to $\Delta \Psi$ collapse, thus appearing unstained in the red channel. (D) Regulation of VDAC1 induced $\Delta \Psi$ collapse by simultaneous overexpression of some apoptotic modulators. The histogram shows the percentage of cells with a collapsed $\Delta \Psi$ measured by flow cytometry in COS-7 cells overexpressing both hVDAC1 (dark gray series) and one of the following proteins: cyclophilin D (Cyp-D), Xiap, Bcl-2 and Bcl-XL. Simultaneous transfections of all these apoptotic modulators with the empty vector pCMS-GFP were used as controls (light gray series). To discard the risk of the toxicity coming from the overexpression at the MOM level, we also transfected a tail-anchored GFP construct targeting the MOM (GFP-MOM) as a control. Bars: SE, * indicates a significant difference compared with the VDAC1 only group $\left(P<0.001, \chi^{2}\right.$-test). (E) Pharmacological modulation of the $\triangle \Psi$ collapse induced by hVDAC1. COS-7 cells transfected with pCMS-GFP-VDAC1-HA were exposed to the following drugs: $50 \mu \mathrm{M}$ zVADfmk, $10 \mu \mathrm{M}$ CsA, $10 \mu \mathrm{M}$ SB203580 or $10 \mathrm{mM} \mathrm{N}$-Acetyl-Cystein (NAC). $\Delta \Psi$ was probed with TMRM and detected by flow cytometry. Bars: SE, * indicates a significant difference compared with the hVDAC1 group $\left(P<0.001, \chi^{2}-\right.$ test). 
A

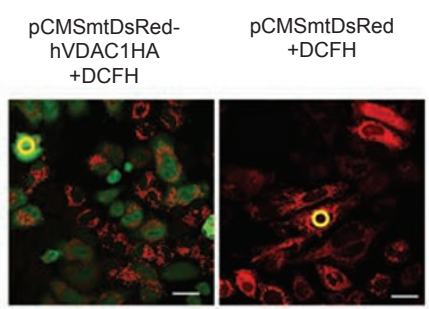

B
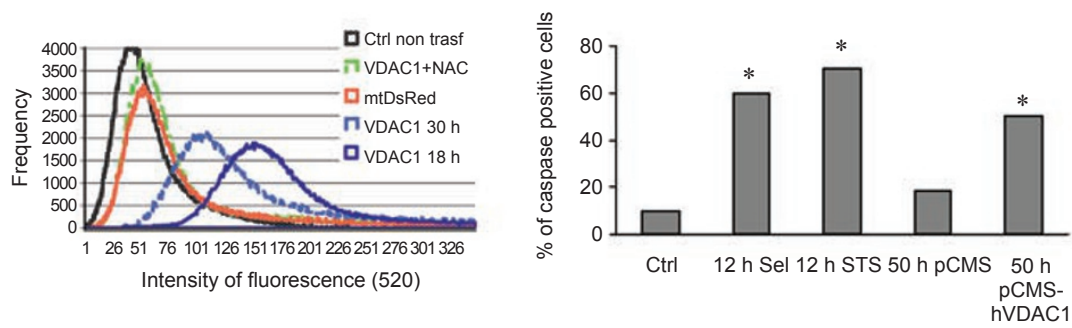

C
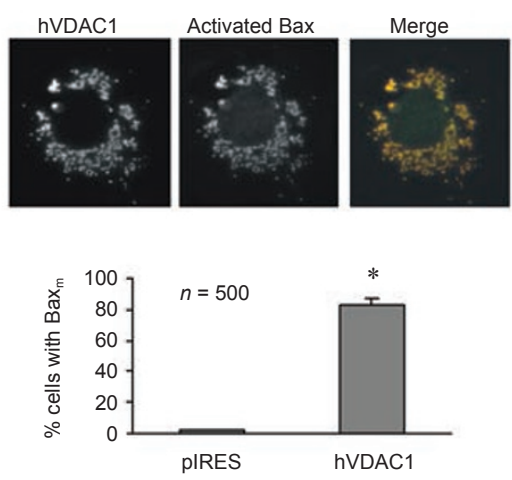

D

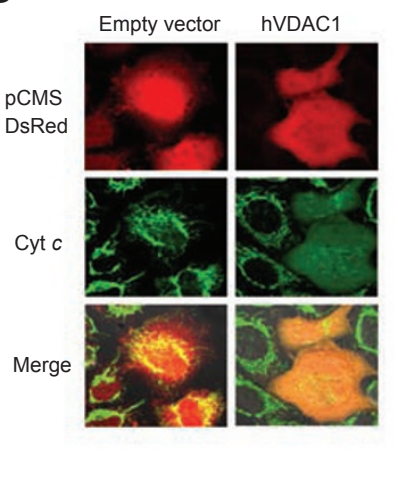

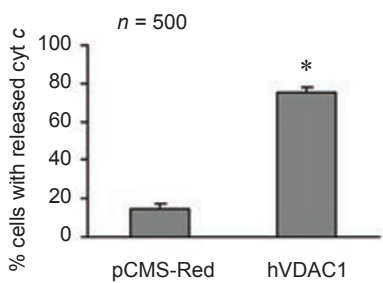

Figure 2 VDAC1 overexpression induces Bax activation, cytochrome $c$ release and caspase cleavage. (A) Intracellular ROS formation in HeLa cells overexpressing hVDAC1. Cells transfected with pCMSmtDsRed-hVADC1 (red) or the empty vector pCMSmtDsRed (red) were loaded with DCFH-DA (green), as described in Materials and Methods. Fluorescence intensity was monitored by imaging on individual cells, $18 \mathrm{~h}$ and $30 \mathrm{~h}$ after transfection. Cells overexpressing hVDAC1, detected by means of the transfection marker mtDsRed were scored for the green signal due to the DCFH oxidation. The treatments with $10 \mathrm{mM}$ NAC completely prevented the increase in green fluorescence associated to the hVDAC1 overexpression. Images were analyzed by drawing regions around individual cells and measuring the value of fluorescence as shown in the chart. Values represent the average of fluorescence measured inside the cell corrected from the basal fluorescence. The results are representative of 30 cells from two independent experiments for each condition. (B) Caspase 3 activity detected in HeLa cells transiently transfected with pCMS-DsRed-hVDAC1 by using the Caspatag ${ }^{\circledR}$ method. The controls used were the following: untreated HeLa cells, HeLa exposed to $20 \mu \mathrm{M}$ selenite or $500 \mathrm{nM}$ staurosporine for $12 \mathrm{~h}$ and HeLa transfected with the empty vector pCMS-DsRed. Fluorescence of the dye was measured by flow cytometry on at least 20000 events for each group. Experiments were done in triplicate. Asterisks indicated a significant difference compared to the untreated or untransfected controls $\left(P<0.001, \chi^{2}\right.$-test). (C) VDAC1 overexpression induces mitochondrial Bax translocation $\left(\right.$ Bax $\left._{\mathrm{m}}\right)$. Upper row: two-color analysis of a HeLa cell transfected with pIRES-VDAC1-HA, fixed and doubly immunostained with a monoclonal antibody against HA (red, VDAC1) and a polyclonal antibody against endogenous activated mitochondrial Bax (green, Bax ${ }_{m}$ ). Lower row: cells were scored for mitochondrial Bax by analysis of multiple fields using Metamorph 4.5 software, and data are presented in the histogram as percentage of cells with mitochondrial Bax among the VDAC1-positive ones. Cells transfected with the empty pIRES plasmid were used as controls. A total of 50 cells were analyzed for each group. Bars: SE, * indicates a significant difference $\left(P<0.001, \chi^{2}\right.$-test). (D) VDAC1 overexpression induces cytochrome $c$ release. The images represent two-color analysis of HeLa cells transfected with pCMS-DsRed-VDAC1-HA or pCMS-DsRed as a control, fixed and immunostained for endogenous cytochrome $c$ (green). Histogram: cells were scored for cytochrome $c$ release by analysis of multiple fields using Metamorph 4.5 software, and data are presented in the histogram as percentage of cells with released cytochrome $c$ among the transfected cells. A total of 50 cells were analyzed for each group. Bars: SE, * indicates a significant difference $\left(P<0.001, \chi^{2}\right.$-test).

MPT triggered by selenite. Indeed, the antioxidant NAC $(10 \mathrm{mM})$ completely inhibited the effect of selenite on
$\Delta \Psi$ in HeLa cells (Figure 3B). Strikingly, pre-incubation with the p38 inhibitor SB203580 also inhibited the $\Delta \Psi$ 
A
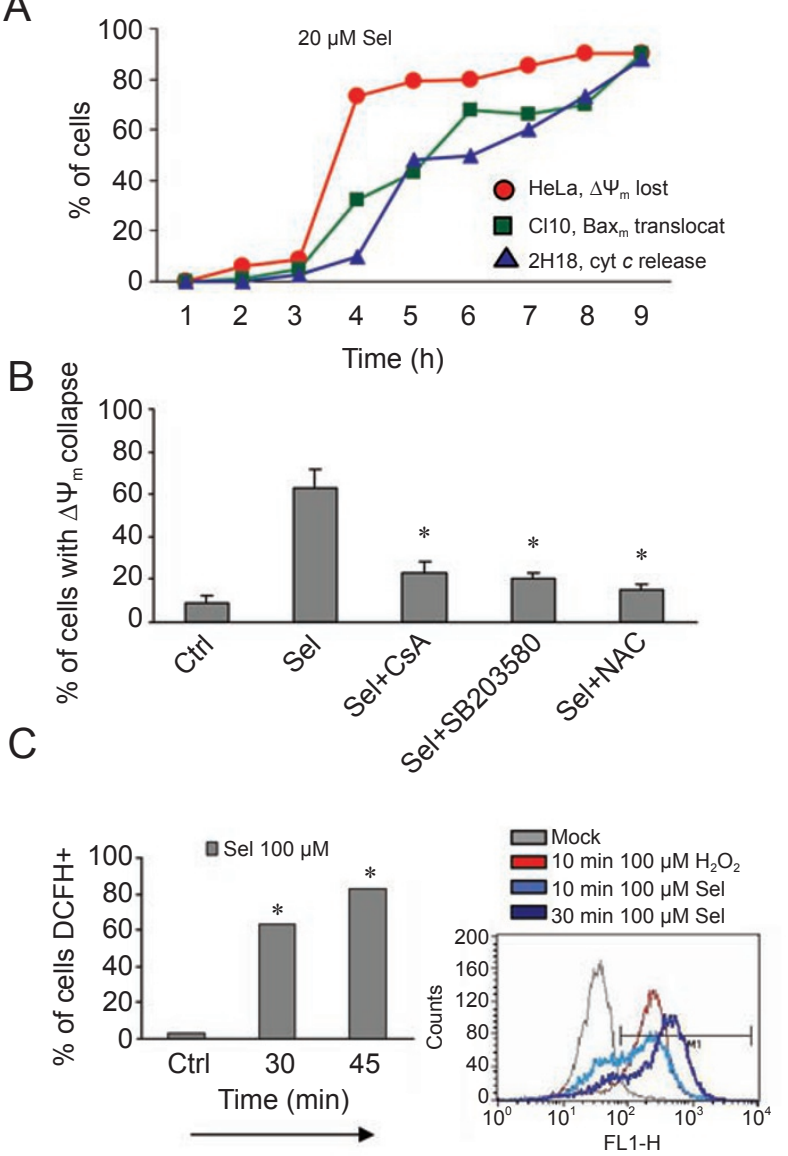

$\mathrm{D}$

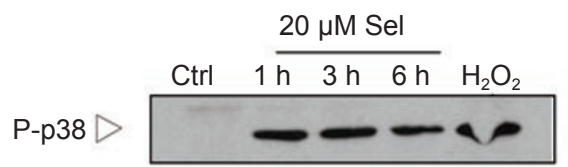

$E$
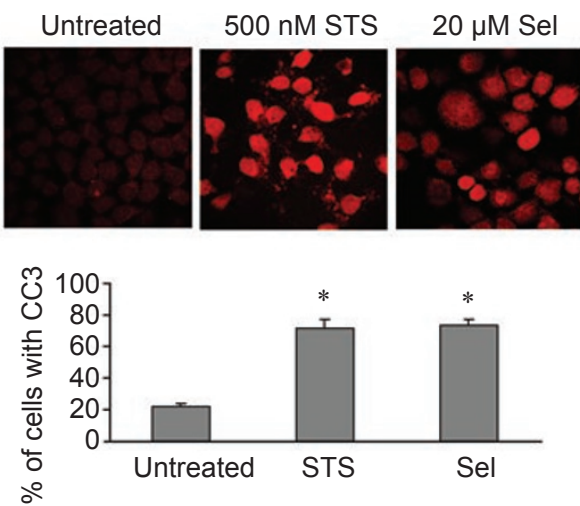

F

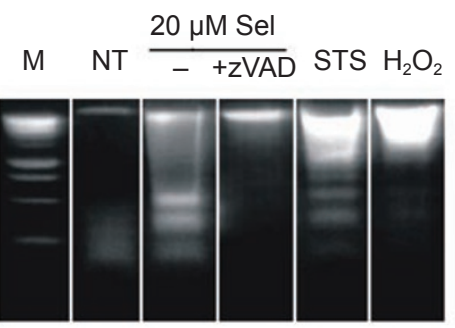

Figure 3 Selenite triggers the PTP in a ROS- and p38-dependent manner and causes apoptotic cell death. (A) Selenite exposure induces $\Delta \Psi$ collapse, Bax activation and cytochrome $c$ release in a time-dependent manner. Time-course measurements of $\Delta \Psi$ loss (TMRM uptake, HeLa cells), Bax activation (GFP-Bax retention, Cl10 cells) and cytochrome $c$ release (Cyt c-GFP retention, $2 \mathrm{H} 18$ cells) were performed following incubation with $20 \mu \mathrm{M}$ selenite (Sel) for the indicated time, by flow cytometry as described in detail in the "Experimental procedures" section. (B) Selenite-induced $\Delta \Psi$ collapse is prevented by CsA, NAC and SB203580. HeLa cells were pretreated with or without $10 \mu \mathrm{M} \mathrm{CsA}, 10 \mathrm{mM}$ NAC or $10 \mu \mathrm{M}$ SB203580 for $1 \mathrm{~h}$ and incubated with $20 \mu \mathrm{M}$ selenite (Sel) for $6 \mathrm{~h}$ and then charged with TMRM to monitor $\Delta \Psi$ by flow cytometry. Bars: SE, * indicates a significant difference compared with the selenite-only group $\left(P<0.001, \chi^{2}\right.$-test). (C) Intracellular ROS generation after selenite exposure. HeLa cells were incubated for the indicated time with $100 \mu \mathrm{M}$ selenite and then loaded with the DCFH-DA dye as described in the "Experimental procedure" section. Untreated cells were used as negative control, while the treatment with $100 \mu \mathrm{M} \mathrm{H}_{2} \mathrm{O}_{2}$ for 15 min was the positive one. Curves in the inserted chart represent the average fluorescence intensity profiles for each population monitored by flow cytometry (FL1). At least 20000 events were scored for each groups. The histogram shows the increase of DCFH fluorescence over the time for selenite-treated cells. Experiments were done in triplicate. Asterisks indicated a significant difference compared to the control $\left(P<0.001, \chi^{2}\right.$-test). (D) p38 activation was monitored by western blotting using a specific anti-Pp38 antibody (phospho-p38) on extracts of HeLa cells incubated with 20 $\mu \mathrm{M}$ selenite for the indicated time. Treatment with $400 \mu \mathrm{M} \mathrm{H}_{2} \mathrm{O}_{2}$ for $1 \mathrm{~h}$ was used as positive control. (E) Caspase activation in response to treatment with $20 \mu \mathrm{M}$ selenite (Sel) for $12 \mathrm{~h}$ was monitored in HeLa cells through immunofluorescence by using a specific antibody raised against the active cleaved form of caspase 3 (CC3). Exposure to 500 nM staurosporine (STS) for $12 \mathrm{~h}$ was used as positive control. The histogram shows the quantitative determination of CC3-positive cell percentage by flow cytometry. Bars: SE, * indicates a significant difference $\left(P<0.001, \chi^{2}\right.$-test). (F) Selenite induces inter-nucleosomal DNA degradation. HeLa cells treated for $15 \mathrm{~h}$ with, $20 \mu \mathrm{M}$ selenite (Sel) in presence or absence of $10 \mu \mathrm{M}$ zVADfmk, $500 \mathrm{nM}$ staurosporine (STS) as positive "apoptotic" control or $400 \mu \mathrm{M} \mathrm{H}_{2} \mathrm{O}_{2}$ as negative "necrotic" control. M, marker; NT, non-treated.

drop caused by selenite (Figure 3B), suggesting that here again p38 was involved in a loop controlling PTP open- ing upon selenite exposure.

Kinetic analysis carried out in HeLa cells revealed that 
the active phosphorylated form of p38 was detectable 1 $\mathrm{h}$ after selenite challenge (Figure 3D). It is noteworthy that this early p38 phosphorylation persisted for up to 6 $\mathrm{h}$ (Figure 3D), while ROS production was transient and rapidly declined after $1 \mathrm{~h}$.

Several mechanisms have been proposed to account for ROS-mediated cell death, including caspase-dependent or -independent apoptosis, as well as necrosis. To unambiguously distinguish between these possibilities, we investigated whether selenite leads to caspase activation.

Confocal microscopy and flow cytometry showed an increase in staining for the cleaved/active form of caspase 3 (in $80 \%$ of HeLa cells $12 \mathrm{~h}$ after selenite addition) (Figure 3E). These results were confirmed by using the caspatag test (Figure 2B). Furthermore, selenite induced DNA laddering that was inhibited by the pan-caspase inhibitor z-VAD-FMK (Figure 3F). This suggests that cell death triggered by selenite involves a canonical apoptotic execution.

\section{Involvement of VDAC1 in the cell death pathway trig- gered by selenite}

Since VDAC1 upregulation is capable of triggering PTP operation committing cells to apoptosis, we reasoned that selenite-mediated PTP activation and subsequent MPT might be prevented by lowering the endogenous levels of VDAC1. Therefore, we silenced VDAC1 in HeLa and C110 cells by RNA interference. Under our experimental conditions, VDAC1 was almost completely suppressed on the sixth day post transfection in both cell lines (Figure 4A). VDAC1 silencing inhibited cell proliferation (not shown) probably because of an impaired ATP-ADP exchange between the cytosol and the mitochondrion [28]. Indeed, in the absence of external stimuli, we detected identical $\Delta \Psi$ values in both control and VDAC1-depleted HeLa cells (Figure 4B). Next, we monitored $\Delta \Psi$ in VDAC1-silenced HeLa cells upon apoptotic stimulation. While selenite treatment induced $\Delta \Psi$ collapse in the control cells, no $\Delta \Psi$ changes were detectable in cells lacking VDAC1. This indicates that VDAC1 is required for PTP opening by selenite. Moreover, VDAC1 silencing also prevented secondary mitochondrial translocation of Bax (C110 cells, Figure 4C) and cyt $c$ release (HeLa cells, Figure 4D). Effect of VDAC1 silencing was specific to selenite, as Bax activation, cyt $c$ release and $\Delta \Psi$ collapse were induced by STS irrespective of VDAC1 levels (Figure 4B, 4C and 4D). These data demonstrate that depletion of VDAC1 not only delays, but completely and selectively prevents, the activation of apoptosis under conditions of oxidative stress caused by selenite. VDAC1 thus seems to be spe- cifically required when apoptosis is triggered as consequence of PTP opening. Correlatively, we observed that (i) upregulating VDAC1 promoted by itself p38 phosphorylation and (ii) that silencing VDAC1 strongly reduced the activation of p38 induced by selenite (Figure 4E).

The inhibitory effect of VDAC1 RNAi on all components of the selenite-induced apoptotic cascade argues for an essential role of VDAC1 within the PTP complex, determining the cell fate during oxidative stress.

\section{Discussion}

\section{VDAC1 overexpression provokes MPT}

Baines et al. [5] recently showed that the three mammalian VDAC isoforms are all dispensable for the induction of MPT, which leads to cell death. This result suggests that VDAC, and especially the VDAC1 isoform, is not a part of the PTP. On the other hand, the literature is crowded of papers reporting a role for VDAC1 in PTPmediated apoptosis $[6,28,30]$. In our opinion, it is difficult to exclude potential compensatory effect in the mice lacking VDAC1 gene. In other words, the comparison of our model of VDAC1 characterization in cellulo and the study of VDAC1 -/- ES cells is truly difficult if not impossible.

In this work, we show that overexpression of VDAC1 triggers $\Delta \Psi$ collapse in living cells. Concomitant overexpression of Cyp-D, which participates in the PTP complex, counteracts this $\Delta \Psi$ collapse. These observations strongly support the notion that VDAC1 is a constituent of the PTP complex at the MOM, as overexpression of VDAC1 in the MOM results in a change in the permeability of the MIM, that is itself regulated by the matrix protein Cyp-D.

Downstream of the $\Delta \Psi$ collapse triggered by VDAC1 overexpression, we detected Bax activation and cyt $c$ release, which are classical hallmarks of the intrinsic pathway of apoptosis. Depending on the apoptotic stimulus, $\Delta \Psi$ collapse can be an early event $[2,48]$, possibly regulated by the Bcl-2 family $[15,49]$ or a late event occuring downstream of caspase activation $[50,51]$. Here, we found that the concomitant overexpression of Bcl-2/ $\mathrm{Bcl}-\mathrm{X}_{\mathrm{L}}$ or treatment with the pan-caspase inhibitor $\mathrm{z}$ VAD-FMK failed to rescue mitochondria from VDAC1induced $\Delta \Psi$ collapse. Moreover $\Delta$ POR1 yeast-strains, which do not express any Bcl-2 family members or caspases, also exhibited $\Delta \Psi$ collapse following exogenous VDAC1 expression (not shown). We conclude that, upon VDAC1 overexpression, $\Delta \Psi$ collapses as a direct consequence of the opening of the PTP complex, which neither depends on Bcl-2 family members nor on caspases. It therefore constitutes in this case an upstream event that 
A

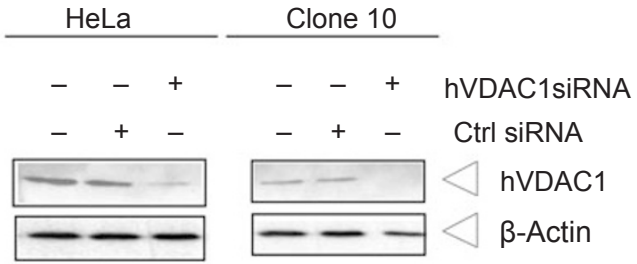

B

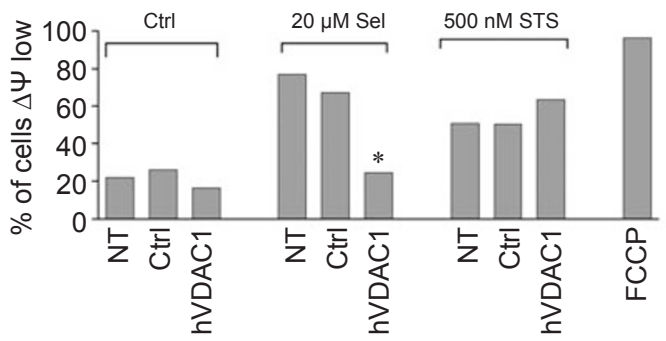

C

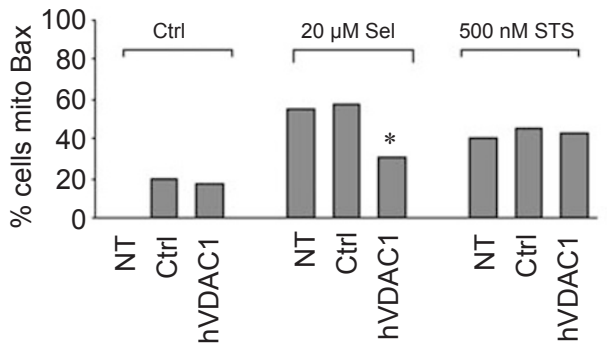

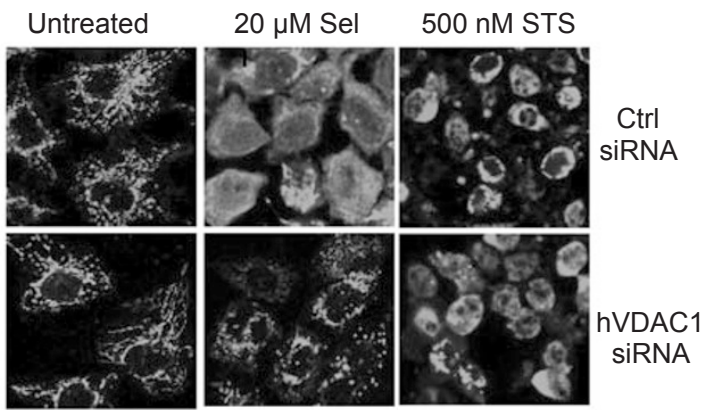

E

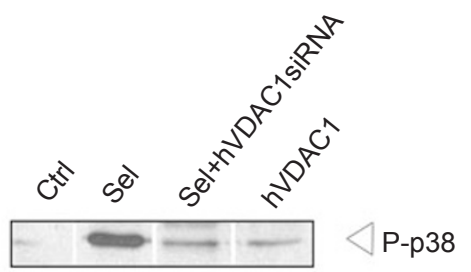

Figure 4 VDAC1 is required for selenite-induced cell death (A) Silencing of VDAC1 expression in HeLa cells or Cl10 cells. Total lysates from HeLa cells or Cl10 cells transfected with specific siRNA for VDAC1 or with scrambled siRNA as control, were immunoblotted by using an anti-VDAC1 antibody. $\beta$-Actin served as loading and transfer efficiency control. (B) Seleniteinduced $\Delta \Psi$ dissipation is prevented by depleting VDAC1. The histogram shows the percentage of cells with a collapsed $\Delta \Psi$ in HeLa cells either non-transfected (NT), transfected with scrambled siRNA (Ctrl) or transfected with siRNAs targeting VDAC1 (hVDAC1). $\triangle \Psi$ was monitored by flow cytometry using TMRM as probe following $6 \mathrm{~h}$ of incubation in the presence or the absence of $20 \mu \mathrm{M}$ selenite (Sel) or $500 \mathrm{nM}$ staurosporine (STS). Treatment with $1 \mu \mathrm{M}$ FCCP served as positive control. Bars: SE, * indicates a significant difference $\left(P<0.001, \chi^{2}\right.$-test). (C) Selenite-induced mitochondrial Bax translocation is reduced in VDAC1-silenced Cl10. The histogram shows Cl10 cells either non-transfected (NT), transfected with scrambled siRNA (Ctrl) or transfected with siRNAs targeting VDAC1. GFP-Bax distribution was monitored by flow cytometry following $6 \mathrm{~h}$ of incubation in the presence or absence of $20 \mu \mathrm{M}$ selenite (Sel) or $500 \mathrm{nM}$ staurosporine (STS). Bars: SE, * indicates a significant difference $\left(P<0.001, \chi^{2}\right.$-test). (D) VDAC1 downregulation abrogates the release of cytochrome $c$ following selenite exposure. Confocal microscopy analysis of HeLa cells transfected with scrambled siRNA or VDAC1-siRNA and incubated in presence or absence of $20 \mu \mathrm{M}$ selenite (Sel) or $500 \mathrm{nM}$ staurosporine (STS) for $6 \mathrm{~h}$ before being processed for immunofluorescence by using an antibody anti-cytochrome $c$. Note that VDAC1 depletion blocks the effect of selenite treatment on $\Delta \Psi_{\mathrm{m}}$ reduction, Bax $\mathrm{m}_{\mathrm{m}}$ translocation and cytochrome $c$ release, but does not affect staurosporine-mediated apoptotic change. (E) VDAC1 depletion reduces selenite-mediated p38 activation, while VDAC1 overexpression activates per se p38. p38 phosphorylation was monitored by western blotting using the anti-Pp38 antibody on the following cell extracts: Ctrl (HeLa untreated), Sel (HeLa exposed to $20 \mu \mathrm{M}$ selenite for $6 \mathrm{~h}$ ), VDAC1-siRNA (HeLa transfected with VDAC1-siRNA and exposed to $20 \mu \mathrm{M}$ selenite for $6 \mathrm{~h}$ ) and VDAC1 (HeLa transfected with pCMS-GFP-VDAC1).

triggers the translocation of Bax, the release of cyt $c$ and the activation of caspases.

VDAC1, ROS and selenite: potential mechanisms of PTP opening

It seems likely that the involvement of VDAC in ap- optosis is not universal but that it rather constitutes an alternative pathway triggered in conditions of oxidative stress. Interestingly, intracellular ROS rises prior to cyt $c$ release during the activation of several apoptotic pathways, in particular those triggered by hyperglycemia, ischemia/reperfusion or viral infection [52-54]. Active 
forms of cell death in yeast and plants also involve ROS, suggesting the existence of an ancestral redox-sensitive death-signaling pathway that is independent of caspases and $\mathrm{Bcl}-2$ [55].

Han et al. [56] showed that VDAC1 is a major escape route for superoxide anions produced by the respiratory chain in mitochondria. In general, VDACs have often been considered as mediators of apoptosis induced by ROS [37, 57-60]. Recently, Simamura et al. [61] demonstrated that the anti-cancer activity of the furanophtoquinone FNQ13 was potentiated by overexpression of VDAC1 and the mitochondrial production of ROS. This finding confirms a previous report from our lab where it was demonstrated that plasma membrane VDAC may act as a transmembrane oxidoreductase using the cytoplasmic NADH as the reducing species [62]. Among candidate chemotherapeutic agents, sodium selenite is one of the most powerful MPT inducers. It reacts directly with cysteine clusters oxidizing the sulfhydryl groups to disulfide linkages, thereby inactivating enzymes [63], and producing both superoxide and peroxide [47]. In this work, we have shown that, following selenite exposure, ROS increase early and activate an apoptotic pathway similar to that induced by VDAC1 overexpression. This cascade is blocked by incubation with the antioxidant NAC. Intriguingly, the p38 MAPK inhibitor SB203580 antagonizes $\Delta \Psi$ collapse induced by either selenite exposure or VDAC1 overexpression, suggesting that an amplification loop exists between the PTP and p38 kinase.

Notably, p38 is involved in several apoptotic pathways mediating Bax activation and translocation [64]. Our work indicates that, following selenite treatment or VDAC 1 overexpression, p38 is phosphorylated. Since ROS induce prolonged MAPK activation by oxidizing/ inactivating the relevant phosphatases [65], the prolonged p38 phosphorylation after selenite exposure or VDAC1 upregulation might result from the oxidative inactivation of $\mathrm{p} 38$ phosphatases.

In summary, we have demonstrated that interference with ROS and p38 pathways protects against apoptosis induced by selenite exposure or VDAC1 upregulation. On this basis, we propose that selenite or VDAC1 raises ROS, leading to phosphorylation of p38. Active P-p38 then exerts positive feedback on VDAC1, thus precipitating $\Delta \psi$ collapse. The mechanism by which $p 38$ regulates VDAC1-dependent MPT might involve a phosphorylation cascade targeting VDAC1 itself or other regulators of the PTP. Accordingly, VDAC1 has been shown to be phosphorylated by $\mathrm{p} 38$ following myocardial ischemia and reperfusion, while the inhibition of $\mathrm{p} 38$, which is cardioprotective, reduces the phosphorylation of VDAC1 [66].
$V D A C 1$ as a selective mediator of the response to oxidative stress

Silencing VDAC1 made cells insensitive to selenite. Consistent with this finding, VDAC1-siRNAs lower the ROS as well as cell death induced by FNQ13 [61], and downregulation of VDAC1 reduces apoptosis induced by cisplatin [67]. Conversely, VDAC1 downregulation does not affect cellular responsiveness to inducers of physiological apoptosis like STS. This finding supports the following two non-exclusive hypotheses: (i) PTP composition may change depending on the stimulus type and (ii) PTP operation may be dispensable in some apoptosis pathways.

VDAC1 depletion not only completely blocked $\Delta \Psi$ collapse upon selenite exposure, but also prevented the entire cell death cascade, as Bax activation, cyt $c$ release and p38 phosphorylation no longer took place. Since selenite-induced cell death mainly depends on ROS generation and PTP opening, we conclude that the assembly of a functional PTP, its opening and the onset of a secondary ROS amplification loop [39] can be achieved only in the presence of VDAC1.

\section{VDAC1 as a therapeutic target}

VDACs are nowadays considered to be promising targets for pro-apoptotic anti-cancer drugs and for antiapoptotic tissue-sparing compounds. Some classic drugs like cisplatin have been shown to be influenced by VDAC [67]. Interestingly, new molecules like FPQ13 [61], methyl jasmonate [68], erastin [60] have been demonstrated to affect the viability of cancer cells through VDACs. Their selectivity for cancer cells is possibly connected to the increase in VDAC expression seen in cancer [61, 69]. In this work, we have demonstrated a role for VDAC in apoptosis induced by oxidative stress. Consistent with this, we and others propose that selenite may be used to specifically target VDAC overexpressing cancers [70]. In addition, the selenite model system described here may be useful to validate drugs targeting this protein of the MOM.

\section{Materials and Methods}

\section{Antibodies and reagents}

Media and sera were from Gibco (Cergy, France). The following drugs were obtained from the sources indicated: sodium selenite, P38 inhibitor (SB203580) and STS from Sigma-Aldrich; NAC and z-VAD-FMK from Calbiochem (La Jolla, CA, USA). Fluorescent probes: TMRM, Hoechst33258 and DCFH-DA were from Molecular Probes (Eugene, Oregon). The antibodies include anti-cyt $c$ (mouse mAb, 1/200; BD Biosciences, San Diego, CA, USA), anti-HA (mouse or chicken Ab; 1/100; Abcam), anti-p-P38 (rabbit pAb; 1/1 000; Ozyme, France), anti-Bax (1/200) (rabbit 
pAb; Santa Cruz, CA, USA), anti-caspase 3 and anti-cleaved caspase 3 (Cell Signalling-1/1 000, Ozyme), anti-actin (rabbit pAb; 1/200; Sigma), and anti-hVDAC1 (rabbit pAb, 1/500, prepared in the laboratory of V De Pinto). The secondary conjugated fluorescent antibodies fluorescein isothiocyanate (FITC) (1/200), Alexa 488 (1/1 000), TexasRed (1/100) and Casacade Blue (1/200) were from Molecular Probes, while the secondary conjugated horseradish peroxidase antibodies $(1 / 5000)$ and the enhanced chemiluminescence (ECL) kit were obtained from Amersham Pharmacia Biotech (Buckinghamshire, UK).

\section{DNA constructs}

The cDNA coding for the isoform 1 of human VDAC tagged with the hemaglutinin epitope (HA) was PCR amplified from pSEYc58-hVDAC1-HA construct (kindly provided by M Forte) using an upper primer incorporating a NheI site and a lower primer with an MluI site. The PCR amplified hVDAC1-HA cDNA was cloned between the NheI and MluI sites of pcDNA3 (Invitrogen, Carlsbad, CA, USA), pIRES, pCMS-GFP and pCMS-DsRed (Clontech).The others constructs used were mtGFP, mtRFP (mt stands for mitochondrial targeting sequence of subunit VIII of citochrome $c$ oxidase) in pCDNA3 (kindly provided by Dr R Rizzuto), EGFP-Bax (kindly provided by Dr RJ Youle) in pEGFP-C3 (Clontech), pCDNA3-GFP-cb5RR (MOM, kindly provided by Dr N Borghese), pCyp-D-CFP, pCFP-Cyp-D (kindly provided by Dr $\mathrm{S}$ Grimm), pcDNA3 Bcl-2 and pcDNA3 $\mathrm{Bcl}-\mathrm{X}_{\mathrm{L}}$.

\section{Cell culture, transfection and treatment}

HeLa (human cervix carcinoma), C110 (HeLa stably transfected with GFP-Bax), 2H18 cells (stable clone of HeLa cells expressing a cyt $c$-GFP fusion protein, kindly provided by Dr D Green), and Cos 7 cells were cultured in Dulbecco's MEM medium (Gibco, Life Technologies) supplemented with $10 \%$ of fetal calf serum (FCS) (Gibco, Invitrogen). The cells were routinely grown as stocks in $75-\mathrm{cm}^{2}$ flasks (Nunc, Polylabo, Strasbourg, France) in a humidified atmosphere $(95 \%$ air, $5 \% \mathrm{CO} 2)$ at $37{ }^{\circ} \mathrm{C}$. The cells were plated into 12 -well plates $\left(1.5 \times 10^{5}\right.$ cells/well $)$ for cytometry, $25-\mathrm{mm}$ round glass coverslips $\left(3 \times 10^{5}\right.$ cells/glass $)$ for confocal microscopy and 60-mm Petri dishes for western blotting $\left(2 \times 10^{6}\right.$ cells/dish). The cells were cultured for $24 \mathrm{~h}$ in the appropriate medium before treatment. G418 was used to maintain stables clones.

Transient transfections with mammalian expression vectors were performed keeping total DNA at $2 \mu \mathrm{g} / \mathrm{ml}$ and cationic Exgene500 in accordance with the protocol provided by the manufacturer. DNAs used for transfection and duration of the experiments are detailed in the figure's legends. To induce apoptosis, transfected or non-transfected cells were washed out by phosphate-buffered saline (PBS), and then either left untreated or treated with the one of following drugs for appropriate time intervals: $500 \mathrm{nM}$ STS (Sigma-Aldrich), $20 \mu \mathrm{M}$ Sel (Sigma-Aldrich) or $400 \mu \mathrm{M} \mathrm{H}_{2} \mathrm{O}_{2}$ (Sigma-Aldrich). When relevant, cells were pre-incubated with inhibitors $(50 \mu \mathrm{M}$ z-VAD-FMK, $10 \mu \mathrm{M}$ CsA, $10 \mu \mathrm{M}$ SB203580, 10 mM NAC) (Sigma-Aldrich) $1 \mathrm{~h}$ before apoptotic stimulation.

\section{siRNA experiments}

Specific silencing of endogenous VDAC1 was achieved by using small inhibitory RNAs (siRNA). Sense and anti-sense oligonucleotides targeting the region 237-257 of the VDAC1 coding sequence (5'-rArgrCrCrArArCrArCUrgrArgrArCrCrArCrCrAr-
ATT-3' and 5'-UUrgrgUrgrgUrCUrCrArgUrgUUrgrgrCUTT-3') were purchased from Genset Proligos. In this region, no homology was observed between VDAC1 and its two other isoforms. Subconfluent HeLa cells or Cl10 cells were transfected, in serum-free Dulbecco's MEM, with $200 \mathrm{nM}(1.7 \mu \mathrm{g})$ of VDAC1-siRNA or non-silencing control siRNA (scrambled sequence, Ambion) using oligofectamine reagent (Invitrogen) according to the manufacturer's procedure. Medium was then supplemented with $20 \%$ FCS, 4 $\mathrm{h}$ after transfection and incubation was continued for another $60-\mathrm{h}$ time lapse before analysis.

\section{Generation of a cell clone expressing GFP-Bax}

HeLa cells grown in $10-\mathrm{cm}$ dishes were transfected with $8 \mu \mathrm{g}$ of pEGFP-Bax plasmid using the standard calcium-phosphate precipitation method. The cells were grown for $24 \mathrm{~h}$ and the medium was replaced. After $72 \mathrm{~h}$, cells containing the above plasmid were selected by adding geneticin (G418-Promega), following previously described protocol [41]. One colony (Cl10) was finally selected, tested and used throughout this study.

\section{Determination of PTP opening}

$\triangle \Psi$ was measured with TMRM (Molecular Probes, Eugene, OR, USA). TMRM accumulates in normal mitochondria due to its positive charge whereby the reduction of $\Delta \Psi$ leads to the release of TMRM. After treatment, adherent cells were washed with PBS and then incubated for $30 \mathrm{~min}$ at $37{ }^{\circ} \mathrm{C}$ with Krebs Ringer Buffered Saline (130 mM NaCl, $3.6 \mathrm{mM} \mathrm{KCl,} 10 \mathrm{mM}$ 4-(2Hydroxyethyl)piperazine-1-ethanesulfonic acid (HEPES), $2 \mathrm{mM}$ $\mathrm{NaHCO}_{3}, 0.5 \mathrm{mM} \mathrm{NaH} \mathrm{PO}_{4}, 0.5 \mathrm{mM} \mathrm{MgCl}, 1.5 \mathrm{mM} \mathrm{CaCl}_{2}, 4.5$ $\mathrm{g} / 1$ glucose, $\mathrm{pH}$ 7.42) supplemented with $200 \mathrm{nM}$ TMRM and 20 $\mu \mathrm{M}$ Verapamil (as a multi drug-resistant pump inhibitor) (Sigma). Cells were then immediately analyzed by microscopy. When flow cytometry analysis was required, cells were detached by short treatment with trypsin-EDTA, resuspended in the above buffer supplemented with $1 \%$ FCS to neutralize the trypsin and immedialtely analyzed on FL3 log mode.

\section{Detection of cyt c release}

Selective permeabilization of HeLa cells with digitonin, followed by immunofluorescence, was used for detection of cyt $c$ release from mitochondria by flow cytometry. Briefly, at the end of the treatments, $10^{7}$ cells were harvested, washed once with PBS and then pelleted. The cell pellet was subsequently incubated for 5 min with $800 \mu$ l of permeabilization buffer $(130 \mathrm{mM} \mathrm{KCl}, 10 \mathrm{mM}$ $\mathrm{NaCl}, 20 \mathrm{mM}$ HEPES, $1 \mathrm{mM} \mathrm{MgSO}$, $5 \mathrm{mM}$ succinic acid, 500 $\mu \mathrm{M}$ EGTA, $50 \mu \mathrm{M}$ Digitonin, $\mathrm{pH}$ 7.2) and then fixed by further incubation with $200 \mu \mathrm{l}$ of $2 \%$ formaldehyde for $1-2 \mathrm{~h}$ at $4{ }^{\circ} \mathrm{C}$. Fixed cells were further permeabilized by 15 min of treatment with $0.2 \%$ Tween at $37{ }^{\circ} \mathrm{C}$, washed, blocked with horse serum and stained by overnight incubation with a specific monoclonal antibody for native cyt $c(1: 200)$ at $4{ }^{\circ} \mathrm{C}$. Stained cells were then washed twice, and finally revealed by $1 \mathrm{~h}$ of incubation with an anti-mouse secondary FITC-conjugated antibody. Immunostained samples were checked by flow cytometry analysis. When relevant, changes in cyt $c$ distribution were monitored in live cells using the same selective permeabilization method on $2 \mathrm{H} 18$ cells [41].

Activated mitochondrial Bax was revealed by flow cytometry detection of $\mathrm{Cl10}$ cells using a selective membrane permeabilization method. After treatments, Cl10 cells were trypsinized and 
each sample was split in two tubes: one was resuspended in Krebs Ringer Buffered Saline, while the other one was permeabilized using the same digitonin buffer described above. In the latter condition, the fluorescence measured was only due to the fraction of GFP-Bax translocated and anchored to the mitochondria, because cytosolic GFP-Bax freely diffuses out form the cell upon digitonin permeabilization. We thus estimated the percentage of Bax translocation for each sample as follows: (number of fluorescent cells in permeabilized sample/number of fluorescent cells in nonpermeabilized sample) $\times 100$. When relevant, Bax translocation from the cytosol to the mitochondria was visualized by confocal microscopy using Cl10 cells. Endogenous mitochondrial-activated Bax in immunostained HeLa or Cos 7 cells was detected by confocal microscopy using specific antibodies against the activated Bax.

Caspase 3 cleavage as well as p38 phosphorylation were assessed by confocal microscopy on fixed HeLa cells processed for indirect immunofluorescence using specific antibodies against the activated forms of these proteins (anti-Phospho-p38 anti-cleaved caspase3, Cell Signalling-Ozyme). Results obtained were confirmed by flow cytometry on fixed and immunostained cells, and/ or by immunoblotting on cell extracts using the same antibodies. Caspase 3 activation was also detected in living cells with the Caspatag $^{\circledR}$ kit (Chemicon), according to the manufacturer's instructions. Cells were then analyzed, measuring the fluorescence emission in FL1 log mode.

\section{Detection of $R O S$}

Intracellular ROS were measured using DCFH-DA (Calbiochem), an oxidation-sensitive fluorescent probe. This test is based on the principle that endogenous esterases hydrolyze DCFH-DA, trapping free DCF inside the cells. ROS, predominantly hydroperoxides, convert non-fluorescent DCF into highly fluorescent DCF. After incubation with drugs, cells were loaded with $20 \mu \mathrm{M}$ DCFHDA for $30 \mathrm{~min}$ at $37^{\circ} \mathrm{C}$ in the dark. Cells were then analyzed on a Becton-Dickinson FACScan (Becton Dickinson) measuring the fluorescence emission in FL1 log mode. The intensity of green fluorescence on individual cells transfected with pCMS-DsRedhVDAC1 was determined over time using the Fluoview Olympus image software (version 1.7).

\section{Flow cytometry}

A total of 20000 cells per sample were analyzed using a PAS Laser/UV flow cytometer (Partec). The cells were excited by an air-cooled argon $488 \mathrm{~nm}$ laser, and then the signal from GFP, CFP, DCFH, FITC or Rhodamine 123 was read on FL1 detector, while the signal from DsRed, Texas Red or TMRM on FL3 detector. The data obtained were acquired, gated, compensated and analyzed using FlowMax software (Partec). Each experiment was repeated at least twice in triplicate.

\section{Indirect immunofluorescence of adherent cells}

After appropriate time of protein-expression or drug-treatment, transfected and non-transfected cells grown on the coverslips were washed with PBS and fixed in $3.7 \%$ formaldehyde for $20 \mathrm{~min}$ at room temperature. Fixed cells were permeabilized using $0.5 \%$ Triton X-100 and unspecific binding was blocked by $30 \mathrm{~min}$ of incubation in $0.2 \%$ gelatine in PBS. Coverslips were incubated in one or a combination of the following anti-sera: rabbit anti-cleaved caspase-3 (1:100), rabbit anti-Pp38 (1:100), mouse anti-HA (1:100), chicken anti-HA (1:100), rabbit anti-ANT2 (1:100), rabbit antiactivated-Bax (1:100) and mouse anti-Cyt $c$ (1:200) in PBS containing $0.2 \%$ gelatin and $1 \%$ Triton $\mathrm{X}-100$ at $4{ }^{\circ} \mathrm{C}$ overnight. After washes in PBS, cells were exposed for $1 \mathrm{~h}$ at TA to the respective anti-mouse or anti-rabbit secondary antibodies conjugated with FITC and/or TexasRed, mounted with Fluoromount-G (Southern Biotechnology, Clinisciences, Montrouge, France) and examined under a fluorescent microscope.

\section{Imaging}

For imaging mitochondrial $\Delta \Psi$, transfected cells were washed with PBS and, when relevant, incubated for $30 \mathrm{~min}$ with $2 \mathrm{nM}$ TMRM (Molecular Probes) and directly mounted in an Attofluor chamber (Atto Instruments) filled with Krebs Ringer Buffered Saline, placed on the imaging stage in a Climacell 2.22 thermostatic device (Cultimat Technologies) set at $37{ }^{\circ} \mathrm{C}$ and observed using a Zeiss Axiovert $100 \mathrm{M}$ inverted motorized microscope equipped of a Micromax 1300 YHS 5 MHz cooled CCD camera (Princetown Instruments). In some cases confocal microscopy was used and cells were imaged using a Zeiss LSM 510 meta CLSM or an Olympus FV1000.

\section{Protein extraction and western blot}

Adherent cells were washed twice in PBS, scraped off with ice-cold lysis buffer $(0.1 \%$ Triton, $0.8 \%$ glycerol, $20 \mathrm{mM}$ Tris$\mathrm{Hcl} \mathrm{pH} 8,137 \mathrm{mM} \mathrm{NaCl}, 1.5 \mathrm{mM} \mathrm{MgCl} 2,1 \mathrm{mM}$ EGTA, $100 \mu \mathrm{M}$ PMSF, $10 \mu \mathrm{g} / \mathrm{ml}$ Leupeptin, $10 \mu \mathrm{g} / \mathrm{ml}$ Aprotinin, $2.5 \mathrm{ng} / \mathrm{ml}$ Pepstatin) and incubated for $1 \mathrm{~h}$ at $4{ }^{\circ} \mathrm{C}$. Lysed cells were further broken using a 26-gauge syringe, and then centrifuged for $10 \mathrm{~min}$ at 10 $000 \times g$. Supernatants were recovered and quantified by the Bradford dye-binding assay (Bio-Rad Laboratories). Equal amounts of protein $(20-50 \mu \mathrm{g})$ for each lysates were size-separated by $10 \%$ $12 \%$ SDS-PAGE and transferred to a Hybond ECL nitrocellulose membrane (Amersham Pharmacia Biotech) using Mini-PROTEAN 3 Cell (Bio-Rad). Membranes were blocked $2 \mathrm{~h}$ in $5 \%$ dry-milk and $0.1 \%$ PBS-Tween at TA, then incubated with relevant primary antibodies (overnight at $4{ }^{\circ} \mathrm{C}$ ), followed by horseradish peroxidase-conjugated secondary antibody ( $1 \mathrm{~h}$ at TA). Immunoreactive bands were detected using ECL reaction (ECL Plus-Amersham Pharmacia) and developed on Kodak films (Sigma).

\section{DNA fragmentation assay}

HeLa cells grown in $100-\mathrm{mm}$ culture dishes $\left(10^{7}\right.$ cells $)$ were collected in their culture medium, pelleted at $800 \times \mathrm{g}$ for $5 \mathrm{~min}$, and washed twice with ice-cold PBS. Pellets were incubated with $1 \mathrm{ml}$ of digestion buffer (100 mM Tris- $\mathrm{HCl} \mathrm{pH} 8.5,0.2 \%$ SDS, $0.1 \%$ Triton, $200 \mathrm{mM} \mathrm{NaCl}, 5 \mathrm{mM}$ EDTA, $100 \mu \mathrm{g} / \mathrm{ml}$ Proteinase $\mathrm{K}$ (Sigma) for $3 \mathrm{~h}$ at $55^{\circ} \mathrm{C}$. Supernatants were collected in a new tube, in which DNA was precipitated with 1 volume of isopropanol and spooned down. Precipitated DNA was washed once with $70 \%$ ethanol, dried up, and resuspended in $30 \mu 1$ of $10 \mathrm{mM}$ Tris$\mathrm{Hcl} 1 \mathrm{mM}$ EDTA. Sample was incubated at $37^{\circ} \mathrm{C}$ for $2 \mathrm{~h}$ with 0.1 $\mathrm{mg} / \mathrm{ml}$ RNAse. DNA fragmentation was visualized with ethidium bromide staining following electrophoresis on a $2 \%$ agar gel.

\section{Statistical analysis}

All data of flow cytometry experiments were representative for at least two sets of independent experiments performed in triplicate and based on at least 20000 events for each group. The 
difference among different groups was examined using $\chi^{2}$-test. A $P$-value below 0.001 was considered to be significant.

\section{Acknowledgements}

This work was supported by l'INSERM, la Région Aquitaine, le Ministère de la Recherche, l'Institut Bergonié, la Ligue Contre le Cancer, le Cancéropole Grand Sud-Ouest, et l'Association France Cancer. VDP thanks FIRB RBRN07BMCT and University of Catania for financial support. We are indebted to Douglas Green for the 2H18 cell clone, Richard Youle for EGFP-Bax, Stephan Grimm for the ANT2 and Cyp-D constructs, Nica Borgese for MOMGFP and Mike Forte for hVDAC1 cDNA. We thank our colleagues Renée Dalibart for technical support and Richard Iggo for reading the manuscript.

\section{References}

1 Bernardi P. Mitochondrial transport of cations: channels, exchangers, and permeability transition. Physiol Rev 1999; 79:1127-1155.

2 De Giorgi F, Lartigue L, Bauer MKA, et al. The permeability transition pore signals apoptosis by directing bax translocation and multimerization. FASEB J 2002; 16:607-609.

3 Zoratti M, Szabò I, De Marchi U. Mitochondrial permeability transitions: how many doors to the house? Biochim Biophys Acta 2005; 1706:40-52.

4 Brenner C, Grimm S. The permeability transition pore complex in cancer cell death. Oncogene 2006; 25:4744-4756.

5 Baines CP, Kaiser RA, Sheiko T, et al. Voltage-dependent anion channels are dispensable for mitochondrial-dependent cell death. Nat Cell Biol 2007; 9:550-555.

6 Galluzzi L, Kroemer G. Mitochondrial apoptosis without VDAC. Nat Cell Biol 2007; 9:487-489.

7 Lemasters JJ. Modulation of mitochondrial membrane permeability in pathogenesis, autophagy and control of metabolism. J Gastroenterol Hepatol 2007; 22 Suppl 1:S31-S37.

8 Colombini M. A candidate for the permeability pathway of the outer mitochondrial membrane. Nature 1979; 279:643645.

9 Benz R. Permeation of hydrophilic solutes through mitochondrial outer membranes: review on mitochondrial porins. Biochim Biophys Acta 1994; 1197:167-196.

10 De Pinto V, Messina A, Accardi R, et al. New functions of an old protein: the eukaryotic porin or voltage dependent anion selective channel (VDAC). Ital J Biochem 2003; 52:17-24.

11 Hiller S, Garces RG, Malia TJ, et al. Solution structure of the integral human membrane protein VDAC-1 in detergent micelles. Science 2008; 321:1206-1210.

12 Bayrhuber M, Meins T, Habeck M, et al. Structure of the human voltage-dependent anion channel. Proc Natl Acad Sci USA 2008; 105:15370-15375.

13 Ujwal R, Cascio D, Colletier JP, et al. The crystal structure of mouse VDAC1 at 2.3 A resolution reveals mechanistic insights into metabolite gating. Proc Natl Acad Sci USA 2008; 105:17742-17747.

14 Rostovtseva T, Colombini M. ATP flux is controlled by a voltage-gated channel from the mitochondrial outer membrane. $J$
Biol Chem 1996; 271:28006-28008.

15 Shimizu S, Narita M, Tsujimoto Y. Bcl-2 family proteins regulate the release of apoptogenic cytochrome $c$ by the mitochondrial channel VDAC. Nature 1999; 399:483-487.

16 Tsujimoto Y, Shimizu S. VDAC regulation by the Bcl-2 family of proteins. Cell Death Differ 2000; 7:1174-1181.

17 Vander Heiden MG, Chandel NS, Li XX, et al. Outer mitochondrial membrane permeability can regulate coupled respiration and cell survival. Proc Natl Acad Sci USA 2000; 97:4666-4671.

18 Kroemer G, Galluzzi L, Brenner C. Mitochondrial membrane permeabilization in cell death. Physiol Rev 2007; 87:99-163.

19 Shi Y, Chen J, Weng C, et al. Identification of the proteinprotein contact site and interaction mode of human VDAC1 with Bcl-2 family proteins. Biochem Biophys Res Commun 2003; 305:989-996.

20 Brdiczka DG, Zorov DB, Sheu S. Mitochondrial contact sites: their role in energy metabolism and apoptosis. Biochim Biophys Acta 2006; 1762:148-163.

21 Fiek C, Benz R, Roos N, et al. Evidence for identity between the hexokinase-binding protein and the mitochondrial porin in the outer membrane of rat liver mitochondria. Biochim Biophys Acta 1982; 688:429-440.

22 Azoulay-Zohar H, Israelson A, Abu-Hamad S, et al. In selfdefence: hexokinase promotes voltage-dependent anion channel closure and prevents mitochondria-mediated apoptotic cell death. Biochem J 2004; 377:347-355.

23 Arzione L, Zilberberg N, Ben-Romano R, Shoshan-Barmatz $\mathrm{V}$. Voltage-dependent anion channel 1-based peptides interact with hexokinase to prevent its anti-apoptotic activity. $J$ Biol Chem 2009; 284:3946-3955.

$24 \mathrm{Xu} \mathrm{X}$, Forbes JG, Colombini M. Actin modulates the gating of neurospora crassa VDAC. J Membr Biol 2001; 180:73-81.

25 Kusano H, Shimizu S, Koya RC, et al. Human gelsolin prevents apoptosis by inhibiting apoptotic mitochondrial changes via closing VDAC. Oncogene 2000; 19:4807-4814.

26 Rostovtseva TK, Bezrukov SM. VDAC regulation: role of cytosolic proteins and mitochondrial lipids. J Bioenerg Biomembr 2008; 40:163-170.

27 Pastorino JG, Hoek JB. Hexokinase II: the integration of energy metabolism and control of apoptosis. Curr Med Chem 2003; 10:1535-1551.

28 Abu-Hamad S, Sivan S, Shoshan-Barmatz V. The expression level of the voltage-dependent anion channel controls life and death of the cell. Proc Natl Acad Sci USA 2006; 103:57875792 .

29 Godbole A, Varghese J, Sarin A, et al. VDAC is a conserved element of death pathways in plant and animal systems. Biochim Biophys Acta 2003; 1642:87-96.

30 De Pinto V, Tomasello F, Messina A, et al. Determination of the conformation of the human VDAC-1 N-terminal peptide, a protein moiety essential for the functional properties of the pore. Chem Biochem 2007; 8:744-756.

31 Lü A, Dong C, Du C, et al. Characterization and expression analysis of paralichthys olivaceus voltage-dependent anion channel (VDAC) gene in response to virus infection. Fish Shellfish Immunol 2007; 23:601-613.

32 Zaid H, Abu-Hamad S, Israelson A, et al. The voltage-dependent anion channel-1 modulates apoptotic cell death. Cell 
Death Differ 2005; 12:751-760.

33 Ganther HE. Selenium metabolism, selenoproteins and mechanisms of cancer prevention: complexities with thioredoxin reductase. Carcinogenesis 1999; 20:1657-1666.

34 Shen HM, Yang CF, Ding WX, et al. Superoxide radical-initiated apoptotic signalling pathway in selenite-treated hepg(2) cells: mitochondria serve as the main target. Free Radic Biol Med 2001; 30:9-21.

35 Shilo S, Aronis A, Komarnitsky R, et al. Selenite sensitizes mitochondrial permeability transition pore opening in vitro and in vivo: a possible mechanism for chemo-protection. Biochem J 2003; 370:283-290.

36 Shen H, Yang C, Liu J, et al. Dual role of glutathione in selenite-induced oxidative stress and apoptosis in human hepatoma cells. Free Radic Biol Med 2000; 28:1115-1124.

37 Madesh M, Hajnóczky G. VDAC-dependent permeabilization of the outer mitochondrial membrane by superoxide induces rapid and massive cytochrome $c$ release. J Cell Biol 2001; 155:1003-1015.

38 Kristal BS, Stavrovskaya IG, Narayanan MV, et al. The mitochondrial permeability transition as a target for neuroprotection. J Bioenerg Biomembr 2004; 36:309-312.

39 Zorov DB, Filburn CR, Klotz LO, et al. Reactive oxygen species (ros)-induced ros release: a new phenomenon accompanying induction of the mitochondrial permeability transition in cardiac myocytes. J Exp Med 2000; 192:1001-1014.

40 Shilo S, Tirosh O. Selenite activates caspase-independent necrotic cell death in Jurkat T cells and J774.2 macrophages by affecting mitochondrial oxidant generation. Antioxid Redox Signal 2003; 5:273-279.

41 Goldstein JC, Waterhouse NJ, Juin P, et al. The coordinate release of cytochrome $c$ during apoptosis is rapid, complete and kinetically invariant. Nat Cell Biol 2000; 2:156-162.

42 Borgese N, Gazzoni I, Barberi M, et al. Targeting of a tailanchored protein to endoplasmic reticulum and mitochondrial outer membrane by independent but competing pathways. Mol Biol Cell 2001; 12:2482-2496.

43 Schubert A, Grimm S. Cyclophilin D, a component of the permeability transition-pore, is an apoptosis repressor. Cancer Res 2004; 64:85-93.

44 Ricci J, Gottlieb RA, Green DR. Caspase-mediated loss of mitochondrial function and generation of reactive oxygen species during apoptosis. J Cell Biol 2003; 160:65-75.

45 Young PR, McLaughlin MM, Kumar S, et al. Pyridinyl imidazole inhibitors of $\mathrm{p} 38$ mitogen-activated protein kinase bind in the ATP site. J Biol Chem 1997; 272:12116-12121.

46 Seko Y, Imura N. Active oxygen generation as a possible mechanism of selenium toxicity. Biomed Environ Sci 1997; 10:333-339.

47 Spallholz JE. On the nature of selenium toxicity and carcinostatic activity. Free Radic Biol Med 1994; 17:45-64.

48 Halestrap AP, McStay GP, Clarke SJ. The permeability transition pore complex: another view. Biochimie 2002; 84:153166.

49 Chipuk JE, Kuwana T, Bouchier-Hayes L, et al. Direct activation of Bax by $\mathrm{p} 53$ mediates mitochondrial membrane permeabilization and apoptosis. Science 2004; 303:1010-1014.

50 Ricci J, Muñoz-Pinedo C, Fitzgerald P, et al. Disruption of mitochondrial function during apoptosis is mediated by cas- pase cleavage of the p75 subunit of Complex I of the electron transport chain. Cell 2004; 117:773-786.

51 Lartigue L, Medina C, Schembri L, et al. An intracellular wave of cytochrome $c$ propagates and precedes bax redistribution during apoptosis. J Cell Sci 2008; 121:3515-3523.

52 Becker LB. New concepts in reactive oxygen species and cardiovascular reperfusion physiology. Cardiovasc Res 2004; 61:461-470.

53 Trinei M, Giorgio M, Cicalese A, et al. A p53-p66shc signalling pathway controls intracellular redox status, levels of oxidation-damaged dna and oxidative stress-induced apoptosis. Oncogene 2002; 21:3872-3878.

54 Sakon S, Xue X, Takekawa M, et al. Nf-kappab inhibits TNFinduced accumulation of ROS that mediate prolonged MAPK activation and necrotic cell death. EMBO J 2003; 22:38983909.

55 Laun P, Pichova A, Madeo F, et al. Aged mother cells of Saccharomyces cerevisiae show markers of oxidative stress and apoptosis. Mol Microbiol 2001; 39:1166-1173.

56 Han D, Antunes F, Canali R, Rettori D, Cadenas E. Voltagedependent anion channels control the release of the superoxide anion from mitochondria to cytosol. J Biol Chem 2003; 278:5557-5563.

57 Petrosillo G, Ruggiero FM, Paradies G. Role of reactive oxygen species and cardiolipin in the release of cytochrome $c$ from mitochondria. FASEB J 2003; 17:2202-2208.

58 Saeed U, Durgadoss L, Valli RK, et al. Knockdown of cytosolic glutaredoxin 1 leads to loss of mitochondrial membrane potential: implication in neurodegenerative diseases. PLoS One 2008; 3:e2459.

59 Veenman L, Shandalov Y, Gavish M. VDAC activation by the $18 \mathrm{kDa}$ translocator protein (TSPO), implications for apoptosis. J Bioenerg Biomembr 2000; 40:199-205.

60 Yagoda N, von Rechenberg M, Zaganjor E, et al. Ras-RafMek-dependent oxidative cell death involving voltage-dependent anion channels. Nature 2007; 447:864-868.

61 Simamura E, Hirai K, Shimada H, et al. Furanonaphthoquinones cause apoptosis of cancer cells by inducing the production of reactive oxygen species by the mitochondrial voltagedependent anion channel. Cancer Biol Ther 2006; 5:15231529.

62 Baker MA, Lane DJR, Ly JD, De Pinto V, Lawen A. VDAC1 is a transplasma membrane NADH-ferricyanide reductase. $J$ Biol Chem 2004; 279:4811-4819.

63 Gopalakrishna R, Gundimeda U. Antioxidant regulation of protein kinase c in cancer prevention. J Nutr 2000; 132:3819S-3823S.

64 Ghatan S, Larner S, Kinoshita Y, et al. P38 Map kinase mediates bax translocation in nitric oxide-induced apoptosis in neurons. J Cell Biol 2000; 150:335-347.

65 Kamata H, Honda S, Maeda S, et al. Reactive oxygen species promote tnfalpha-induced death and sustained jnk activation by inhibiting map kinase phosphatases. Cell 2005; 120:649661.

66 Schwertz H, Carter JM, Abdudureheman M, et al. Myocardial ischemia/reperfusion causes VDAC phosphorylation which is reduced by cardioprotection with a p38 map kinase inhibitor. Proteomics 2007; 7:4579-4588.

67 Tajeddine N, Galluzzi L, Kepp O, et al. Hierarchical involve- 
ment of Bak, VDAC1 and Bax in cisplatin-induced cell death. Oncogene 2008; 27:4221-4232.

68 Goldin N, Arzoine L, Heyfets A, et al. Methyl jasmonate binds to and detaches mitochondria-bound hexokinase. Oncogene 2008; 27:4636-4643.

69 Shinohara Y, Ishida T, Hino M, et al. Characterization of porin isoforms expressed in tumor cells. Eur J Biochem 2000; 267:6067-6073.

70 Simamura E, Shimada H, Hatta T, et al. Mitochondrial voltage-dependent anion channels (VDACs) as novel pharmacological targets for anti-cancer agents. J Bioenerg Biomembr 2008; 40:213-217. 